\title{
Human enteroids: preclinical models of non-inflammatory diarrhea
}

\author{
Olga Kovbasnjuk ${ }^{+1}$, Nicholas C Zachos ${ }^{\dagger 1}$, Julie In', Jennifer Foulke-Abel', Khalil Ettayebi², Joseph M Hyser², \\ James R Broughman², Xi-Lei Zeng ${ }^{2}$, Sabine Middendorp², Hugo R de Jonge ${ }^{4}$, Mary K Estes*2 and Mark Donowitz*1
}

\begin{abstract}
Researchers need an available and easy-to-use model of the human intestine to better understand human intestinal physiology and pathophysiology of diseases, and to offer an enhanced platform for developing drug therapy. Our work employs human enteroids derived from each of the major intestinal sections to advance understanding of several diarrheal diseases, including those caused by cholera, rotavirus and enterohemorrhagic Escherichia coli. An enteroid bank is being established to facilitate comparison of segmental, developmental, and regulatory differences in transport proteins that can influence therapy efficacy. Basic characterization of major ion transport protein expression, localization and function in the human enteroid model sets the stage to study the effects of enteric infection at the transport level, as well as to monitor potential responses to pharmacological intervention.
\end{abstract}

\section{Introduction}

The goal of our project has been to establish human organoid/enteroid cultures as a preclinical model to study the pathophysiology of non-inflammatory (small intestinal) diarrheas and inflammatory diarrhea models as well as to develop drug therapy. The rate-limiting step in translational research is often the absence of reliable preclinical models that adequately reflect relevant human physiology and disease pathophysiology. Moreover, drug development based on in vitro cell culture and/or animal models often fails in clinical trials due to ineffectiveness

${ }^{\dagger}$ Contributed equally

*Correspondence: mdonowit@jhmi.edu; mestes@bcm.edu

'Department of Medicine, Division of Gastroenterology, Johns Hopkins University School of Medicine, 720 Rutland Avenue, Baltimore, MD 21205, USA

2Department of Molecular Virology and Microbiology, Baylor College of Medicine, One Baylor Plaza, Houston, TX 77030, USA

Full list of author information is available at the end of the article and/or significant side effects. Since detailed studies of the contributions of specific transport proteins to normal intestinal water and electrolyte homeostasis have not been accomplished in human intestine, one cause of these failures that must be considered is that differences may exist between humans and other model systems in the expression, localization and/or regulation of proteins that are important for normal intestinal transport or that become abnormal as part of the pathophysiology of diarrhea. There is thus an urgent need to develop and validate novel preclinical models of human diseases in general.

This need is particularly true in the field of human diarrheal diseases, which cause $\sim 4 \%$ of all deaths worldwide, including 1.2 million deaths in children per year, and are a leading cause of death in the aged population in the USA [1]. The morbidity/mortality caused by diarrhea is due to abnormal regulation of ion and water transport across the intestinal epithelium leading to significant dehydration. The goal of therapy is to rehydrate the patient either using transport processes not affected by the diarrheal disease (the concept on which oral rehydration solutions were developed) or by restoring normal intestinal electrolyte transport. In all diarrheal diseases there is reduced $\mathrm{Na}^{+}$absorption primarily due to inhibition of the brush border $\mathrm{Na}^{+} / \mathrm{H}^{+}$ exchanger NHE3 and perhaps the $\mathrm{Cl}^{-} / \mathrm{HCO}_{3}{ }^{-}$exchanger DRA, while in enterotoxigenic diarrheas there is additional stimulation of anion secretion via the cystic fibrosis transmembrane conductance regulator (CFTR) $[2,3]$. Unfortunately, over the past 35 years the approaches to correct this abnormal intestinal ion transport in diarrhea have failed to produce any significant drug for treating diarrhea to supplement the use of oral rehydration solution-based therapy, which is currently used in only $\sim 33 \%$ of children with severe diarrhea in developing countries [1].

\section{Approach}

A human intestinal model that duplicates normal physiologic salt and water transport and is reproducible and readily available is needed as part of the strategy for drug 
development for diarrheal diseases. In developing such a model, consideration must be given to segmental differences along the horizontal axis of the gastrointestinal tract that relate to different transport proteins being represented, as well as to regulation of those transporters by the local neuroendocrine environment, which is relevant to regulation of multiple transport processes [4]. Components of the microbiome that are present luminally also are likely to influence this regulation. Transport proteins are attractive drug targets that likely require consideration of developmental stages, so a system to duplicate differences in transport protein expression across infant, adult, and aged populations would be useful. Another potential barrier for drug development in diarrheal diseases relates to the presence of polymorphisms, epigenetic modifications and differences in drug metabolism in individual patients, an area that has led to the concept of personalized medicine.

Recent progress in the identification and isolation of human intestinal epithelial stem cells has led to the creation ex vivo of three-dimensional small intestinal epithelial functional units called intestinal organoids and enteroids that include the entire villus-crypt axis and all epithelial cell types normally present. Two techniques using human intestinal stem cells have been reported [5-7]. One starts with human pluripotent stem cells (H9 or others; WiCell International Stem Cell Bank, Madison, WI, USA) that differentiate into organoid cultures which contain multiple cell types, including enterocytes, goblet cells, enteroendocrine cells, Paneth cells and mesenchymal cell populations. The second technique starts with whole intestinal crypts isolated from adult human intestinal tissue (surgery or biopsies) and includes only epithelial cells, Paneth cells, goblet cells and enteroendocrine cells (expression of $\mathrm{M}$ cells requires additional culture conditions [8]) but does not contain mesenchymal elements. The development of these enteroids was pioneered by the ground breaking studies of Hans Clevers and his coworkers in Utrecht, the Netherlands, who identified the intestinal stem cell as one expressing Lgr5 [9] and established the conditions needed to initiate small and large intestinal enteroid growth and long-term culture, as well as the conditions to induce differentiation $[5,10]$. The approaches to grow enteroids are very recent and modifications have been reported [11-13].

Using Clevers' methods, we have been establishing human enteroids as a model to understand the physiologic control of intestinal water and electrolyte transport, including changes that occur in digestion and become exaggerated in diarrheal diseases. Recently, Dekkers and colleagues demonstrated in human enteroids derived from rectal biopsies that forskolin-induced luminal dilation can serve as a functional assay to measure CFTR function [14]. Models presently established in our laboratories include enteroids from the human duodenum, jejunum, ileum and proximal colon. Studies have been initiated to characterize them as regards: polarity; expression of transport proteins involved in intestinal $\mathrm{Na}^{+}$absorption and $\mathrm{Cl}^{-}$secretion, including expression along the horizontal axis of the intestine and along the villus-crypt axes under normal conditions and in disease; comparison with intact human tissue concerning localization of transport proteins and polarization markers along vertical and horizontal axes of the intestine; ability to freeze these ex vivo preparations as well as to re-establish the cultures from the frozen specimens; maintenance of functional characteristics over multiple passages; interaction with luminal bacteria and viruses as components of the human microbiome; and genetic and epigenetic diversity.

The enteroids were obtained from endoscopic biopsies from subjects felt to have no organic intestinal pathology after endoscopy and surgical specimens that otherwise would have been discarded, which is enabling establishment of an enteroid bank. This will facilitate consideration of biologic diversity among human subjects in terms of all parameters to be studied. These enteroids are polarized, based on localization of known apical (villin), basolateral membrane $\left(\mathrm{Na}^{+} / \mathrm{K}^{+}\right.$-ATPase $)$markers, and tight junction morphology using immunofluorescence and transmission electron microscopy (Figure 1). The enteroids express transport proteins that take part in $\mathrm{Na}^{+}$ absorption and anion secretion similar to normal human intestine along the horizontal and vertical axes of the intestine. NHE3, DRA, SGLT1 and CFTR are expressed apically, while $\mathrm{Na}^{+} / \mathrm{K}^{+}$-ATPase and NKCC1 are localized to the basolateral membrane; SGLT1 is present in the duodenum and jejunum, minimally expressed in the ileum, and absent in the proximal colon. The enteroids also express $\mathrm{Ca}^{2+}$-activated $\mathrm{Cl}^{-}$channels and $\mathrm{K}^{+}$channels. Although enteroids do not form flat monolayers in this particular culture system, ion transport activity can be measured in intact three-dimensional cultures. Regulation of $\mathrm{Na}^{+}$absorption and $\mathrm{Cl}^{-}$secretion in the enteroids occur as in human intestine, with forskolin inhibiting NHE3 and stimulating $\mathrm{Cl}^{-}$secretion by activating CFTR (Figure 2). This regulation mimics what occurs in the human jejunum in the early postprandial period.

Besides their relevance for understanding human intestinal physiology, the enteroids serve as an excellent model of human enteric infections. We previously showed that organoids are susceptible to infection with rotavirus [15] and we have now evaluated the use of enteroids to study rotavirus infectivity and replication. Jejunal enteroids established from tissues of patients undergoing bariatric surgery can support rotavirus replication, as confirmed by detection of nonstructural viral proteins by immunofluorescence (Figure 3a) and increased levels of viral RNA 

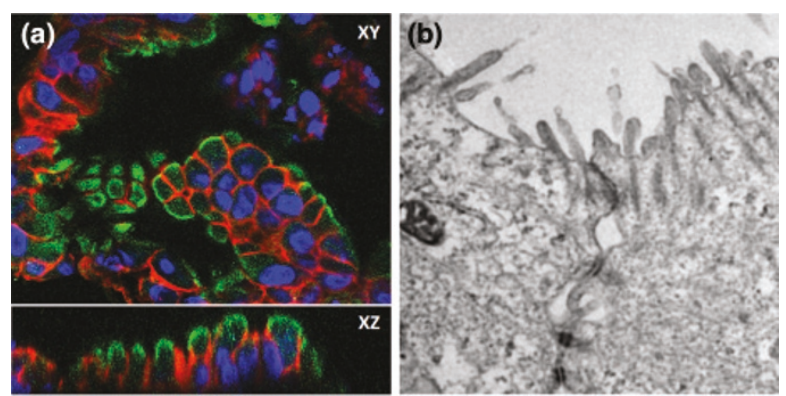

Figure 1. Cells within human enteroids reflect morphology of native intestinal tissue. (a) Human enteroids are well polarized, expressing apical villin (green) and basolateral $\mathrm{Na}^{+} / \mathrm{K}^{+}$-ATPase (red). Nuclei are indicated by Hoescht 33342 (blue). (b) Transmission electron microscopy demonstrates presence of intact tight junctions. $n=3$ for each experiment.
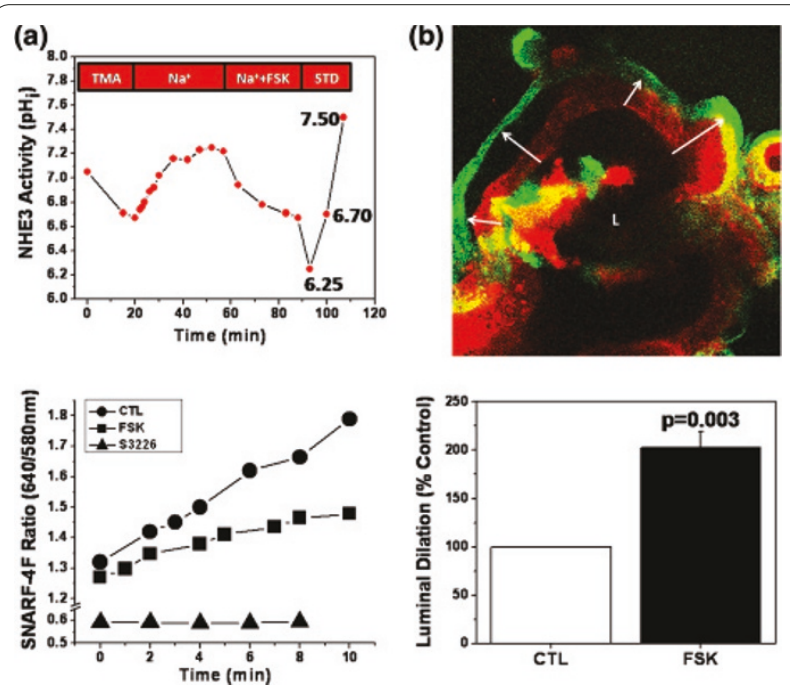

Figure 2. Human enteroids recapitulate two major functions of the small intestine: $\mathrm{Na}^{+}$absorption and $\mathrm{Cl}^{-}$secretion.

(a) Enteroids exhibit NHE3 activity, which can be inhibited by forskolin (FSK) treatment before or after initiation of transport measurements. Differentiated enteroids were loaded with the pH-sensitive dye SNARF-4F and prepulsed with $50 \mathrm{mM} \mathrm{NH}_{4} \mathrm{Cl}$ to acidify the cytosol. $\mathrm{Na}^{+}$-dependent alkalinization in the presence of $50 \mu \mathrm{M}$ HOE694 is due to NHE3 activity (no alkalinization in the presence of the NHE3 inhibitor S3226, added after HOE694). $n=6$ for each condition. CTL, control; STD, pH standards; TMA, tetramethylammonium chloride. (b) Duodenal enteroid lumens (L) significantly dilate in response to elevated cAMP levels via FSK treatment. After FSK treatment, the size of enteroid lumens increased $203 \pm 16 \%$ over controls. The same optical section of enteroids loaded with SNARF-4F was compared before (red) and after (green) 30 minutes of FSK treatment. Arrows indicate change in position of epithelial layer due to luminal dilation. $n=3$ for each experiment.

by quantitative RT-PCR (Figure 3b). The nonstructural viral proteins, NSP4 and NSP5, show the expected punctate staining patterns, representing viroplasms, the sites of virus replication and particle assembly. These

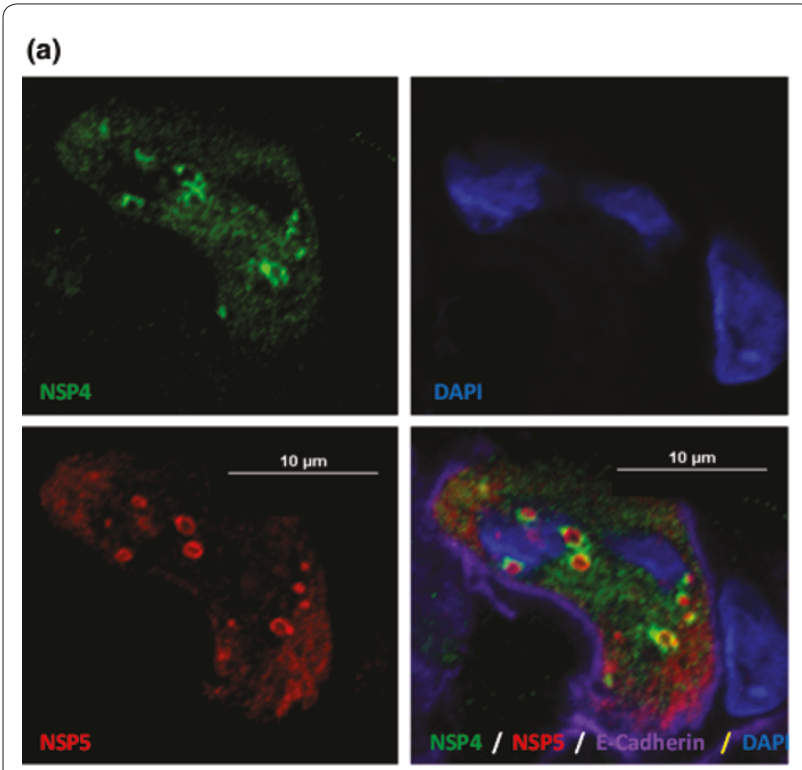

(b)

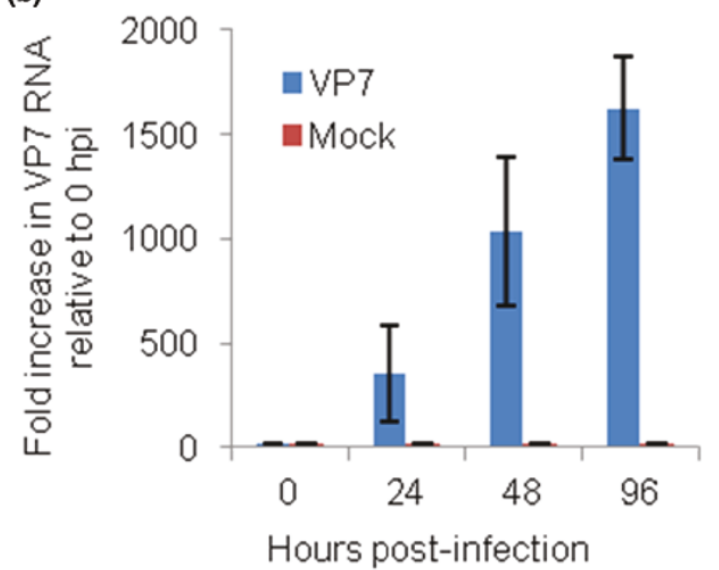

Figure 3. Human jejunal enteroids support rotavirus growth. (a) Nonstructural viral proteins, NSP4 (green) and NSP5 (red), were detected in enteroids by confocal microscopy. Basolateral membrane marked by E-cadherin (purple) and nuclei (blue) by 4,6-diamidino2-phenylindole (DAPI) staining. (b) GAPDH-normalized rotavirus VP7 RNA levels are shown as fold increases relative to 0 hours post infection. Values are means of triplicates and standard deviation for three amplifications per sample.

results demonstrate the ability to compare rotavirus infection and replication in organoids and enteroids, and demonstrate that the enteroids may be useful to study pathogenesis of multiple enteric pathogens.

\section{Conclusion}

These studies have been initiated recently, and while enteroid culture and initial functional studies have been achieved, further goals in the study of pathophysiology of diarrhea must consider two factors. First, one must consider the pathogenesis of diarrhea in neural regulation of intestinal $\mathrm{Na}^{+}$absorption and $\mathrm{Cl}^{-}$secretion, given that 
$\sim 50 \%$ of several severe diarrheas (cholera toxin-induced and rotavirus diarrhea) are neurally mediated $[16,17]$. The second factor to consider is the role of differentiation of the enteroids in the expression level and localization of transport proteins and their physiologic and pathophysiologic regulation. Future studies will also evaluate changes in expression of key transport proteins following infection as well as establishing methods to evaluate drug effects on normal and infected cultures, including those grown as monolayers [18].

\section{Abbreviations}

CFTR, cystic fibrosis transmembrane conductance regulator; PCR, polymerase chain reaction; $\mathrm{RT}$, reverse transcriptase.

\section{Competing interests}

The authors declare that they have no competing interests.

\section{Acknowledgements}

This work was supported in part by National Institutes of Health NCATS and NIDDK Grants U18-TR000552 (NCATS), R01-DK26523, R01-DK61765, P01-DK072084, K08-DK088950, R01-Al080656 and P30-DK56336 (Hopkins), and P30-DK089502 (Baylor). The authors acknowledge use of the Kudsi Imaging Core Facility of the Conte Hopkins Digestive Disease Basic \& Translational Research Core Center. This study was approved by the Johns Hopkins University School of Medicine and Baylor College of Medicine Institutional Review Boards. The publication costs for this article were funded through NIH NCATS grant U18-TR000552.

\section{Declarations}

Publication of this supplement has not been supported by sponsorship. Articles have undergone the journal's standard review process. The Editors declare that they have no competing interests.

This article has been published as part of Stem Cell Research \& Therapy Volume 4 Supplement 1, 2013: Stem cells on bioengineered microphysiological platforms for disease modeling and drug testing. The full contents of the supplement are available online at http://www.stemcellres.com/supplements/4/S1.

\section{Author details}

'Department of Medicine, Division of Gastroenterology, Johns Hopkins University School of Medicine, 720 Rutland Avenue, Baltimore, MD 21205 USA. ${ }^{2}$ Department of Molecular Virology and Microbiology, Baylor College of Medicine, One Baylor Plaza, Houston, TX 77030, USA. ${ }^{3}$ Department of Pediatric Gastroenterology, Wilhelmina Children's Hospital, University Medical Center Utrecht, Lundlaan 6, 3584 EA Utrecht, the Netherlands. ${ }^{4}$ Department of Gastroenterology \& Hepatology, Erasmus University Medical Center, s'Gravendijkwal 230, 3000 CA Rotterdam, the Netherlands.

\section{Published: 20 December 2013}

\section{References}

1. Donowitz M, Alpers DH, Binder HJ, Brewer T, Carrington J, Grey MJ: Translational approaches for pharmacotherapy development for acute diarrhea. Gastroenterology 2012, 142:e1-e9.

2. Abreu MT, Arnold ET, Chow JYC, Barrett KE: Phosphatidylinositol 3-kinasedependent pathways oppose Fas-induced apoptosis and limit chloride secretion in human intestinal epithelial cells: implications for inflammatory diarrheal states. J Biol Chem 2001, 276:47563-47574.
3. Petri WA, Miller M, Binder HJ, Levine MM, Dillingham R, Guerrant RL: Enteric infections, diarrhea, and their impact on function and development. J Clin Investig 2008, 118:1277-1290.

4. Gershon MD: Genes and lineages in the formation of the enteric nervous system. Curr Opin Neurobiol 1997, 7:101-109.

5. Sato T, Stange DE, Ferrante M, Vries RGJ, van Es JH, van den Brink S, van Houdt WJ, Pronk A, van Gorp J, Siersema PD, Clevers H: Long-term expansion of epithelial organoids from human colon, adenoma, adenocarcinoma, and Barrett's epithelium. Gastroenterology 2011, 141:1762-1772.

6. Spence JR, Mayhew CN, Rankin SA, Kuhar MF, Vallance JE, Tolle K, Hoskins EE, Kalinichenko VV, Wells SI, Zorn AM, Shroyer NF, Wells JM: Directed differentiation of human pluripotent stem cells into intestinal tissue in vitro. Nature 2011, 470:105-109.

7. Jung P, Sato T, Merlos-Suárez A, Barriga FM, Iglesias M, Rossell D, Auer H, Gallardo M, Blasco MA, Sancho E, Clevers H, Batlle E: Isolation and in vitro expansion of human colonic stem cells. Nat Med 2011, 17:1225-1227.

8. de Lau W, Kujala P, Schneeberger K, Middendorp S, Li VSW, Barker N, Martens A, Hofhuis F, DeKoter RP, Peters PJ, Nieuwenhuis E, Clevers H: Peyer's patch M cells derived from $\mathrm{Lgr} 5^{+}$stem cells require SpiB and are induced by RankL in cultured 'miniguts'. Mo/ Cell Biol 2012, 32:3639-3647.

9. Barker N, van Es JH, Kuipers J, Kujala P, van den Born M, Cozijnsen M, Haegebarth A, Korving J, Begthel H, Peters PJ, Clevers H: Identification of stem cells in small intestine and colon by marker gene Lgr5. Nature 2007, 449:1003-1007.

10. Sato T, Vries RG, Snippert HJ, van de Wetering M, Barker N, Stange DE, van Es $\mathrm{JH}$, Abo A, Kujala P, Peters PJ, Clevers H: Single Lgr5 stem cells build cryptvillus structures in vitro without a mesenchymal niche. Nature 2009 459:262-265

11. Jabaji Z, Sears CM, Brinkley GJ, Lei NY, Joshi VS, Wang J, Lewis M, Stelzner M, Martín MG, Dunn JCY: Use of collagen gel as an alternative extracellular matrix for the in vitro and in vivo growth of murine small intestinal epithelium Tissue Eng Part C Methods 2013. doi:10.1089/ten.tec.2012.0710 [Epub ahead of print]

12. Lahar N, Lei NY, Wang J, Jabaji Z, Tung SC, Joshi V, Lewis M, Stelzner M, Martín MG, Dunn JCY: Intestinal subepithelial myofibroblasts support in vitro and in vivo growth of human small intestinal epithelium. PLoS One 2011, 6:e26898.

13. Miyoshi H, Ajima R, Luo CT, Yamaguchi TP, Stappenbeck TS: Wnt5a potentiates TGF- $\beta$ signaling to promote colonic crypt regeneration after tissue injury. Science 2012, 338:108-113.

14. Dekkers JF, Wiegerinck CL, de Jonge HR, Bronsveld I, Janssens HM, de Winterde Groot KM, Brandsma AM, de Jong NWM, Bijvelds MJC, Scholte BJ, Nieuwenhuis EES, van den Brink S, Clevers H, van der Ent CK, Middendorp S, Beekman JM: A functional CFTR assay using primary cystic fibrosis intestinal organoids. Nat Med 2013, 19:939-945

15. Finkbeiner $S R$, Zeng XL, Utama B, Atmar RL, Shroyer NF, Estes MK: Stem cellderived human intestinal organoids as an infection model for rotaviruses. mBio 2012, 3:e00159-12.

16. Lundgren $0: 5$-Hydroxytryptamine, enterotoxins, and intestinal fluid secretion. Gastroenterology 1998, 115:1009-1012.

17. Lundgren O, Peregrin AT, Persson K, Kordasti S, Uhnoo I, Svensson L: Role of the enteric nervous system in the fluid and electrolyte secretion of rotavirus diarrhea. Science 2000, 287:491-495.

18. Sato T, Clevers $\mathrm{H}$ : Growing self-organizing mini-guts from a single intestinal stem cell: mechanism and applications. Science 2013, 340:1190-1194.

\section{doi:10.1186/scrt364}

Cite this article as: Kovbasnjuk O, et al:: Human enteroids: preclinical models of non-inflammatory diarrhea. Stem Cell Research \& Therapy 2013, 4(Suppl 1):S3. 